\title{
Two-dimensional discrete solitons in rotating lattices
}

\author{
Jesús Cuevas, ${ }^{1}$ Boris A. Malomed, ${ }^{2}$ and P. G. Kevrekidis ${ }^{3}$ \\ ${ }^{1}$ Grupo de Física No Lineal, Departamento de Física Aplicada I, Escuela Universitaria Politécnica, \\ C/ Virgen de África, 7, 41011 Sevilla, Spain \\ ${ }^{2}$ Department of Physical Electronics, School of Electrical Engineering, Faculty of Engineering, \\ Tel Aviv University, Tel Aviv 69978, Israel \\ ${ }^{3}$ Department of Mathematics and Statistics, University of Massachusetts, Amherst, Massachusetts 01003-4515, USA
}

(Received 15 May 2007; published 19 October 2007)

\begin{abstract}
We introduce a two-dimensional discrete nonlinear Schrödinger (DNLS) equation with self-attractive cubic nonlinearity in a rotating reference frame. The model applies to a Bose-Einstein condensate stirred by a rotating strong optical lattice, or light propagation in a twisted bundle of nonlinear fibers. Two types of localized states are constructed: off-axis fundamental solitons (FSs), placed at distance $R$ from the rotation pivot, and on-axis $(R=0)$ vortex solitons (VSs), with vorticities $S=1$ and 2. At a fixed value of rotation frequency $\Omega$, a stability interval for the FSs is found in terms of the lattice coupling constant $C, 0<C$ $<C_{\mathrm{cr}}(R)$, with monotonically decreasing $C_{\mathrm{cr}}(R)$. VSs with $S=1$ have a stability interval, $\tilde{C}_{\mathrm{cr}}^{(S=1)}(\Omega)<C$ $<C_{\mathrm{cr}}^{(S=1)}(\Omega)$, which exists for $\Omega$ below a certain critical value, $\Omega_{\mathrm{cr}}^{(S=1)}$. This implies that the VSs with $S=1$ are destabilized in the weak-coupling limit by the rotation. On the contrary, VSs with $S=2$, that are known to be unstable in the standard DNLS equation, with $\Omega=0$, are stabilized by the rotation in region $0<C<C_{\mathrm{cr}}^{(S=2)}$, with $C_{\mathrm{cr}}^{(S=2)}$ growing as a function of $\Omega$. Quadrupole and octupole on-axis solitons are considered too, their stability regions being weakly affected by $\Omega \neq 0$.
\end{abstract}

DOI: 10.1103/PhysRevE.76.046608

PACS number(s): 05.45.Yv, 03.75.Lm, 42.65.Tg, 42.70.Qs

\section{INTRODUCTION}

Discrete dynamical systems represented by nonlinear lattices in one, two, and three dimensions constitute a class of models which are of fundamental interest by themselves, and, simultaneously, they find applications of paramount importance in various fields of physics. One such example is known in nonlinear optics, where the one-dimensional (1D) discrete nonlinear Schrödinger (DNLS) equation was predicted by Christodoulides and Joseph to support fundamental discrete solitons [1] with an even profile. Such nonlinear structures were later created experimentally in an array of semiconductor waveguides [2]. Subsequently, stable odd solitons, alias twisted localized modes, were predicted and studied in detail in the same model [3], as well as in an array of photorefractive waveguides with photovoltaic nonlinearity [4].

Another experimental realization of dynamical lattices in the optical domain is possible in photorefractive crystals, where a quasidiscrete setting can be induced by counterpropagating laser beams illuminating the crystal in the normal polarization, while the probe beam, which can sustain solitary waves, is launched in the extraordinary polarization. The difference from the array of waveguides fabricated in silica or in a semiconductor material is that the photorefractive nonlinearity is saturable, rather than cubic. This method of the creation of photonic lattices was proposed in Ref. [5], and results obtained by means of the technique were reviewed in Ref. [6]. In particular, both fundamental and twisted solitons in the 1D lattice were reported in Ref. [7], and fundamental solitons (FSs) in the 2D lattice were created too [8], as well as 2D vortex solitons (VSs) in the same setting [9].

Recently, the progress in the technology of writing permanent arrays of channels in silica slabs has made it possible to create 2D waveguiding lattices with the cubic nonlinearity, which emulate a bundle of nonlinear optical fibers with linear coupling in the transverse plane [10]. In particular, spatial lattice solitons of the surface and corner types were reported in this setting [11] (surface solitons were reported too in the 2D photonic lattice in a photorefractive crystal [12]). Thus, 1D and 2D discrete dynamical models have a potential for further applications to nonlinear optical media of various types.

Another natural realization for the lattice systems is provided by a Bose-Einstein condensate (BEC) trapped in an optical lattice (OL). If the OL is strong enough, the underlying Gross-Pitaevskii equation (GPE) for the wave function in the continuum may be approximated by its DNLS counterpart $[13,14]$; the relevance of the discrete model in this setting has also been demonstrated experimentally in 1D by testing its predictions experimentally (see, e.g., Ref. [15]). Higher-dimensional OLs can be easily created too [16]. Thus, the relevant DNLS equation may be one, two, or three dimensional; in particular, various species of 3D discrete solitons, including those with intrinsic vorticity, have been predicted in this setting [17], and their stability has been systematically analyzed [18]. Still another implementation of the DNLS lattice in the space of any dimension, from one to three, is possible in terms of a crystal of microcavities trapping photons [19] or polaritons [20].

Discrete solitons of various kinds have been studied in detail theoretically in $1 \mathrm{D}, 2 \mathrm{D}$, and $3 \mathrm{D}$ versions of the DNLS equation (see an earlier review [21] and the more recent works mentioned above). As mentioned above, some of these solitons have been created experimentally in optical media equipped with fabricated or photoinduced lattices. All these localized states have their counterparts in continuum models with periodic potentials that emulate the lattices. In particu- 
lar, 2D solitons of both the fundamental and vortex types, which are unstable in uniform continua with the cubic selffocusing nonlinearity, can be readily stabilized by the periodic OL potential [22]; for the stabilization of FSs, a quasi-1D potential is sufficient, instead of its full 2D counterpart [23]. Vortices are unstable too in the uniform space with the saturable nonlinearity [24], in which case they can also be stabilized by the periodic potential $[22,9]$. The relevance of these stabilization mechanisms in the continuum was also demonstrated for higher-order vortex solitons, and so-called supervortices, i.e., arrays of compact vortices with global vorticity imprinted onto the array, under both cubic and saturable nonlinearities [25].

Recently, it was shown that 2D solitons obeying the GPE in the $2 \mathrm{D}$ continuum can also be supported by a rotating $\mathrm{OL}$ $[26,27]$. These solitons may be fully localized (spot-shaped) solutions to the equation with the self-focusing or attractive cubic nonlinearity, placed at some distance from the rotation pivot and revolving in sync with the holding 2D lattice. In particular, the soliton can be placed at a local minimum of the rotating potential, while the pivot is set at a local maximum. These co-rotating strongly localized solitons are stable provided that the rotation frequency $\Omega$ does not exceed a critical value $\left(\Omega_{\mathrm{cr}}\right)_{\min }$. In the same model, but with a rapidly rotating OL, stable ring-shaped solitons (with zero vorticity), i.e., objects localized along the radius but delocalized in the azimuthal direction, have been found too, for $\Omega$ exceeding another critical value $\left(\Omega_{\mathrm{cr}}\right)_{\max }$. Note that the model does not support any stable pattern in interval $\left(\Omega_{\mathrm{cr}}\right)_{\min }<\Omega$ $<\left(\Omega_{\mathrm{cr}}\right)_{\max }$ [26]. On the other hand, stable ring-shaped states, with both zero and nonzero vorticity, have been found in the model with the repulsive cubic nonlinearity and rotating quasi-one-dimensional (periodic) potential, if $\Omega$ exceeds a respective critical value. Obviously, the latter model does not give rise to any localized state in the absence of the rotation.

A rotating OL can be easily implemented in BEC experiments [28]; then, if the OL is strong enough, it is natural to approximate the GPE in the co-rotating reference frame by an appropriate variety of the 2D DNLS equation. In addition to that, such a model may also describe the light propagation in a twisted bundle of nonlinear optical fibers, linearly coupled in the transverse plane by tunneling of light between the fiber cores. The objective of the present work is to introduce a model of the rotating discrete lattice, and find stable discrete solitons in it, both FSs and VSs.

The paper is organized as follows. In Sec. II, we formulate the model, taking the underlying GPE in the reference frame co-rotating with the OL, and replacing the continuum equation by its discrete version corresponding to a strong periodic potential. Discrete FSs are considered in Sec. III. We construct the solutions starting from the anticontinuum limit, which corresponds to the zero value of the coupling constant accounting for the linear interaction between neighboring sites of the discrete lattice, $C=0$. A family of FS solutions is constructed by continuation in $C$; their stability is examined by the computation of eigenfrequencies for infinitesimal perturbations around the soliton, and verified by direct simulations of the evolution of perturbed FSs. It is found that the FS, with its center located at distance $R$ from the rotation pivot, is stable within an interval $0<C<C_{\mathrm{cr}}(R)$, with $C_{\text {cr }}$ decaying as a function of $R$. Section III also includes a simple analytical approximation, which makes it possible to explain the decrease of $C_{\text {cr }}$ with the growth of $R$. In Sec. IV, we consider localized vortices (VSs), whose center coincides with the rotation pivot. For the VS with vorticity $S=1$, the stability region is found to be $\widetilde{C}_{\mathrm{cr}}^{(S=1)}<C$ $<C_{\mathrm{cr}}^{(S=1)}$, provided that the rotation frequency $\Omega$ is smaller than a critical value $\Omega_{\mathrm{cr}}$ (the stability interval shrinks to nil at $\Omega=\Omega_{\mathrm{cr}}$ ). Vortices with $S=2$ are considered too. While in the ordinary (nonrotating) DNLS model, with $\Omega=0$, all VSs of the latter type are unstable [29], we demonstrate that the rotation opens a stability window for them, $0<C<C_{\mathrm{cr}}^{(S=2)}$, with $C_{\mathrm{cr}}^{(S=2)}$ growing as a function of $\Omega$. Direct numerical simulations are also used to illustrate the dynamical evolution of FSs and VSs when they are unstable. Results obtained in this work and related open problems are summarized in Sec. V.

\section{MODEL}

The starting point is the normalized 2D GPE, which includes the potential in the form of an OL rotating at angular velocity $\Omega$, and thus stirring a "pancake"-shaped (quasi-flat) Bose-Einstein condensate (BEC) trapped in a narrow gap between two strongly repelling optical sheets. Unlike the analysis performed in Refs. [26,27], where simulations were run in the laboratory reference frame, here we write the GPE in the reference frame co-rotating with the lattice, hence the potential does not contain explicit time dependence:

$$
\begin{aligned}
i \frac{\partial \psi}{\partial t}= & -\left(\frac{1}{2} \nabla^{2}+\Omega \hat{L}_{z}\right) \psi-\epsilon[\cos (k(x-\xi))+\cos (k(y-v))] \psi \\
& +\sigma|\psi|^{2} \psi .
\end{aligned}
$$

Here, $\hat{L}_{z}=i\left(x \partial_{y}-y \partial_{x}\right) \equiv i \partial_{\theta}$ is the operator of the $z$ component of the orbital momentum ( $\theta$ is the polar angle), and $\sigma$ determines the sign of the interaction, attractive $(\sigma=-1)$ or repulsive $(\sigma=+1)$. Constants $\xi$ and $v$ determine a possible shift of the lattice with respect to the rotation pivot. Note that Eq. (1) does not contain any additional trapping potential, as we are interested in solutions localized under the action of the OL.

It is more convenient to shift the origin of the Cartesian coordinates to a lattice node, thus replacing Eq. (1) by the translated form

$$
\begin{aligned}
i \frac{\partial \psi}{\partial t}= & -\left[\frac{1}{2} \nabla^{2}+i \Omega\left[(x+\xi) \partial_{y}-(y+v) \partial_{x}\right]\right] \psi \\
& -\epsilon[\cos (k x)+\cos (k y)] \psi+\sigma|\psi|^{2} \psi .
\end{aligned}
$$

As shown in a general form in Ref. [14], a discrete model, which corresponds to the limit of a very deep OL, can be derived from the underlying GPE in the tight-binding approximation. Eventually, it amounts to a straightforward discretization of the GPE. Thus, the discrete counterpart of Eq. (2) is 


$$
\begin{aligned}
i \frac{d \psi_{m, n}}{d t}= & -\frac{C}{2}\left\{\left(\psi_{m+1, n}+\psi_{m-1, n}+\psi_{m, n+1}+\psi_{m, n-1}-4 \psi_{m, n}\right)\right. \\
& -i \Omega\left[(m+\xi)\left(\psi_{m, n+1}-\psi_{m, n-1}\right)\right. \\
& \left.\left.-(n+v)\left(\psi_{m+1, n}-\psi_{m-1, n}\right)\right]\right\}+\sigma\left|\psi_{m, n}\right|^{2} \psi_{m, n}
\end{aligned}
$$

where $(m, n)$ are discrete coordinates, and $C>0$ is the corresponding coupling constant, which accounts for the linear tunneling of atoms between BEC droplets trapped in deep nodes of the lattice. As mentioned above, Eq. (3) may also describe a twisted bundle of nonlinear optical fibers linearly coupled by the tunneling of light in the transverse plane, $(m, n)$. In that case, $t$ is the propagation distance along the fiber, and only $\sigma=-1$, i.e., the attractive or focusing nonlinearity, is the relevant choice.

Equation (3) conserves the norm and Hamiltonian,

$$
\begin{gathered}
N=\sum_{m, n}\left|\psi_{m, n}\right|^{2} \\
H=\sum_{m, n}\left\{\frac{C}{2}\left(\left|\psi_{m+1, n}-\psi_{m, n}\right|^{2}+\left|\psi_{m, n+1}-\psi_{m, n}\right|^{2}\right)+\frac{1}{2} \sigma\left|\psi_{m, n}\right|^{4}\right. \\
-\frac{i C}{4} \Omega\left[( m + \xi ) \left(\psi_{m, n}^{*}\left(\psi_{m, n+1}-\psi_{m, n-1}\right)\right.\right. \\
\left.-\psi_{m, n}\left(\psi_{m, n+1}^{*}-\psi_{m, n-1}^{*}\right)\right)-(n+v)\left(\psi_{m, n}^{*}\left(\psi_{m+1, n}-\psi_{m-1, n}\right)\right. \\
\left.\left.\left.-\psi_{m, n}\left(\psi_{m+1, n}^{*}-\psi_{m-1, n}^{*}\right)\right)\right]\right\} .
\end{gathered}
$$

In addition to $C$, the discrete model contains three irreducible parameters: $\Omega$, which takes values $0<\Omega<\infty$, and the coordinates of the pivot displacement, $(\xi, v)$, which take values $0 \leq \xi, v<1$, plus the sign parameter, $\sigma= \pm 1$. As in the usual 2D DNLS equation (with $\Omega=0$ ), the values $\sigma= \pm 1$ in Eq. (3) may be transformed into each other by the staggering transformation $\psi_{m, n} \rightarrow(-1)^{m+n} \psi_{m, n}$, therefore we fix $\sigma \equiv-1$ (selfattraction).

Our first objective is to find stationary localized solutions to Eq. (3) in the form of FSs and VSs. To this end, we substitute the standing wave ansatz $\psi_{m, n}=e^{i \Lambda t} \phi_{m, n}$, where $-\Lambda$ is the normalized chemical potential, in terms of the underlying BEC model; then, the stationary lattice field $\phi_{m, n}$ obeys the equation

$$
\begin{aligned}
\Lambda \phi_{m, n}= & \frac{C}{2}\left(\phi_{m+1, n}+\phi_{m-1, n}+\phi_{m, n+1}+\phi_{m, n-1}-4 \phi_{m, n}\right) \\
& +i \frac{C}{2} \Omega\left[(m+\xi)\left(\phi_{m, n+1}-\phi_{m, n-1}\right)\right. \\
& \left.-(n+v)\left(\phi_{m+1, n}-\phi_{m-1, n}\right)\right]+\left|\phi_{m, n}\right|^{2} \phi_{m, n} .
\end{aligned}
$$

Note that solutions for $\phi_{m, n}$ are complex, unless $\Omega=0$. The second objective will be to examine the stability of the discrete solitons, assuming small perturbations in the form of $\delta \psi_{m, n} \sim \exp (i \Lambda t+i \lambda t)$, the onset of instability indicated by the emergence of $\operatorname{Im}(\lambda) \neq 0$. The evolution of unstable solitons will be examined by means of direct simulations of Eq. (3).
To parametrize the soliton families, we fix the scales by setting $\Lambda \equiv 1$; obviously, with $C>0$, only positive $\Lambda$ may give rise to localized solutions, while $C$ will be varied.

\section{FUNDAMENTAL SOLITONS}

The rotation makes the discrete lattice inhomogeneous, hence the properties of solitons strongly depend on the location of their centers. Without the rotation, Eq. (3) amounts to the ordinary 2D DNLS equation, in which various species of discrete solitons and their stability have been studied in detail. In particular, FSs, which are represented by real solutions, are stable at $C \leq C_{\mathrm{cr}}=2 \Lambda \equiv 2[21]$. The onset of their instability is accounted for by a pair of eigenfrequencies of small perturbations with finite imaginary and zero real parts, i.e., the instability leads to the exponential growth of perturbations. Accordingly, numerical simulations of the instability development demonstrate spontaneous transformation of unstable FSs into lattice breathers [30]. In this section, we first report numerical results obtained for the stability of FSs in the model with $\Omega \neq 0$, and then present an analytical estimate that may explain numerical findings.

\section{A. Numerical results}

Our analysis aimed to determine the stability border for the FSs, $C_{\mathrm{cr}}$, for each set of values of the discrete coordinates of the soliton's center, $\left\{m_{0}, n_{0}\right\}$. Here we present results for angular velocity $\Omega=0.1$ and zero pivot displacement $\xi=v$ $=0$ [in Ref. [26], the rotation pivot was fixed at a local maximum of the potential in Eq. (2), which corresponds to setting $\xi=v=1 / 2$ in Eqs. (3) and (6), and the center of the soliton trapped in the lattice was placed at a local minimum closest to the pivot, which would mean $\left.\left\{m_{0}, n_{0}\right\}=\{0,0\}\right]$. This choice makes it possible to explore the existence and stability of FSs in a clear form, while larger values of $\Omega$ give rise to a resonance with linear lattice modes, leading to Wannier-Stark ladders and hybrid solitons [33] and making the continuation in $C$ and identification of $C_{\text {cr }}$ difficult. We carried out the calculations on the lattice of size $21 \times 21$, since for this case, the lattice was for all the considered cases much wider than the very localized FS structures of interest. To avoid effects of the boundaries, the range of the soliton center's coordinates was restricted to $\left|m_{0}\right|,\left|n_{0}\right| \leq 8$.

The FS solutions were looked for starting at point $C=0$, i.e., at the so-called anticontinuum limit [21]. In this limit, the FS is seeded by using a nonzero value of the field at a single point, the center of the FS, $\phi_{m, n}^{(C=0)}=\delta_{m, m_{0}} \delta_{n, n_{0}}$; obviously, this expression satisfies Eq. (6) with $\Lambda=1$ and $C=0$. After the branch of the FS solutions had been found by the continuation in $C$, its stability was quantified through the computation of eigenfrequencies of small perturbations, using the equation linearized about the FS.

Figure 1 displays a typical example of the thus found dependence of eigenfrequencies $\lambda$ of small perturbations on the lattice coupling constant [as said above, the instability corresponds to $\operatorname{Im}(\lambda) \neq 0]$, for the FS with its center set at point $\left(m_{0}=3, n_{0}=2\right)$; for comparison, the dependence of the instability growth rate, i.e., $|\operatorname{Im}(\lambda)|$, on $C$ is also shown for 

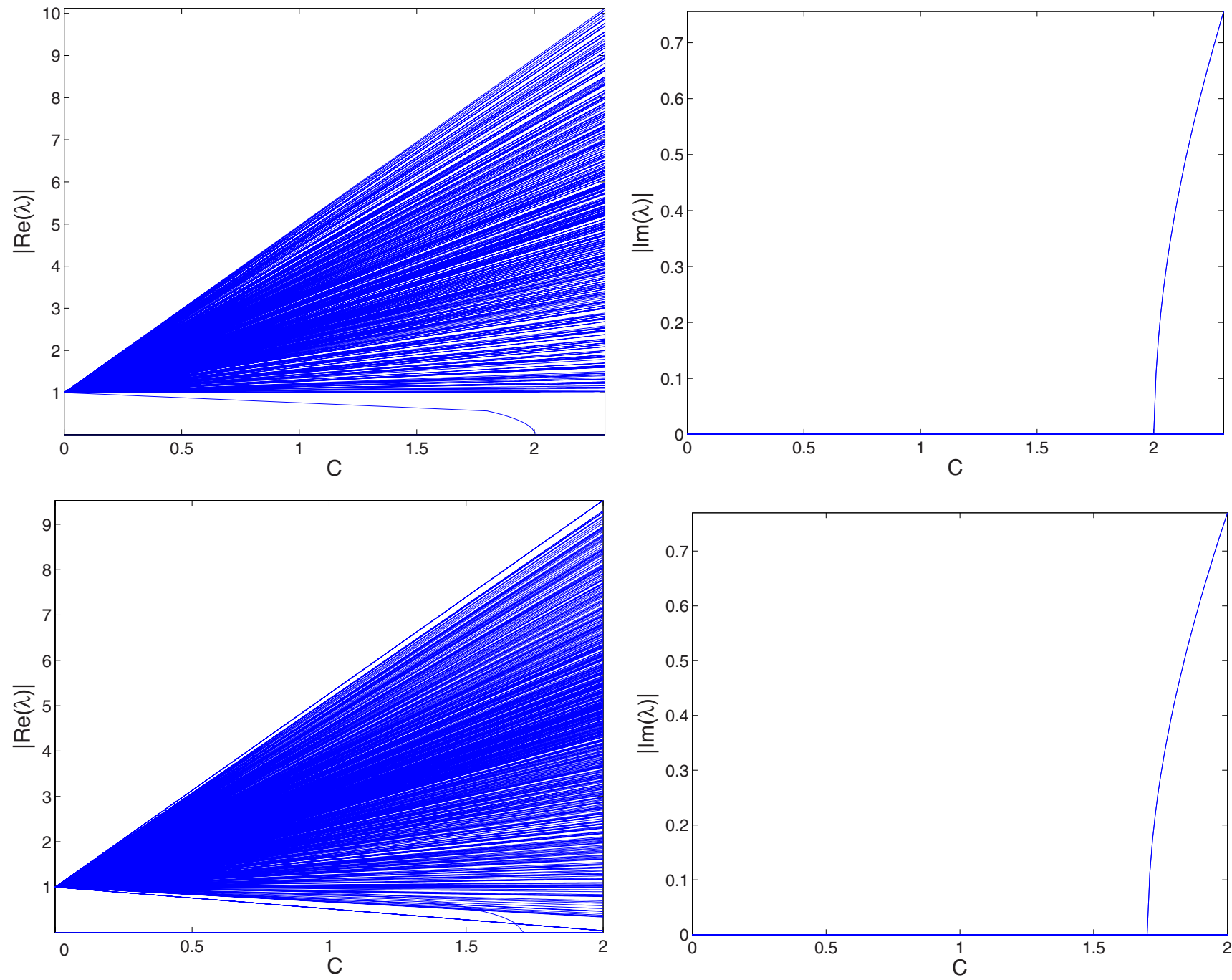

FIG. 1. (Color online) Plots of real and imaginary parts (left and right panels) of the eigenfrequencies of linearization around fundamental solitons in the ordinary nonrotating lattice, with $\Omega=0$ (top panels), and for their counterparts in the present model (with $\Omega=0.1$ ). In the latter case, the soliton is centered at $\left(m_{0}=3, n_{0}=2\right)$.

the FS in the usual DNLS model $(\Omega=0)$ in the top panel of the figure. It is seen that the instability sets in at $C=C_{\mathrm{cr}}$ $=1.70$, which is smaller than the critical value in the ordinary model $C_{\mathrm{cr}}^{(\Omega=0)}=2$. Typical examples of stable and unstable FSs belonging to the family presented in Fig. 1 are displayed in Figs. 2 and 3.

Results obtained for the FSs placed at different positions are summarized in Fig. 4, in the form of dependences of $C_{\mathrm{cr}}$ on the distance of the FS's center from the pivot, $R$ $\equiv \sqrt{m_{0}^{2}+n_{0}^{2}}$, and on one coordinate $n_{0}$, while $m_{0}$ is fixed. It is observed that $C_{\mathrm{cr}}$ monotonously decreases with $R$, starting from $C_{\mathrm{cr}}=2$ at $R=0$ (we recall again that $C_{\mathrm{cr}}^{(\Omega=0)}=2$ for the FS in the ordinary DNLS equation). Note that there are different pairs $\left(m_{0}, n_{0}\right)$ which have equal values of $R=\sqrt{m_{0}^{2}+n_{0}^{2}}$ and give slightly different $C_{\mathrm{cr}}$. For instance, for the pair $(5,0)$, $C_{\mathrm{cr}}=1.51$, while $C_{\mathrm{cr}}=1.50$ for $(4,3)$. Hence, the stability depends on the two-dimensional structure of the solution.

Figures 1 and 3 show that the FS is destabilized, with the increase of $C$, through the appearance of a pair of imaginary eigenfrequencies. Direct simulations of the dynamical evolu- tion of unstable FSs in the framework of Eq. (3) demonstrate that the instability does not destroy the solitary wave. Instead, it transforms the waveform into a persistent breathing structure (see a typical example in Fig. 5).

\section{B. Semi-analytical estimates}

The decrease of $C_{\mathrm{cr}}$ with the increase of the distance of the FS from the pivot $R$, which is the main feature revealed by the above numerical analysis, as shown in Fig. 4, can be explained using an estimate based on the quasicontinuum approximation. To this end, we note that stationary solutions to the underlying continuum equation (1) are looked for as $\psi=e^{i \Lambda t} \phi(x, y)$, with the function $\phi$ obeying the stationary equation

$$
\Lambda \phi=-C\left(\frac{1}{2} \nabla^{2}+\Omega \hat{L}_{z}\right) \phi-\epsilon[\cos (k x)+\cos (k y)] \Phi-\Phi^{3} .
$$

Here, following the discrete model, we have set $\xi=v=0, \sigma$ $=-1$, and explicitly introduced the spatial-scale parameter 

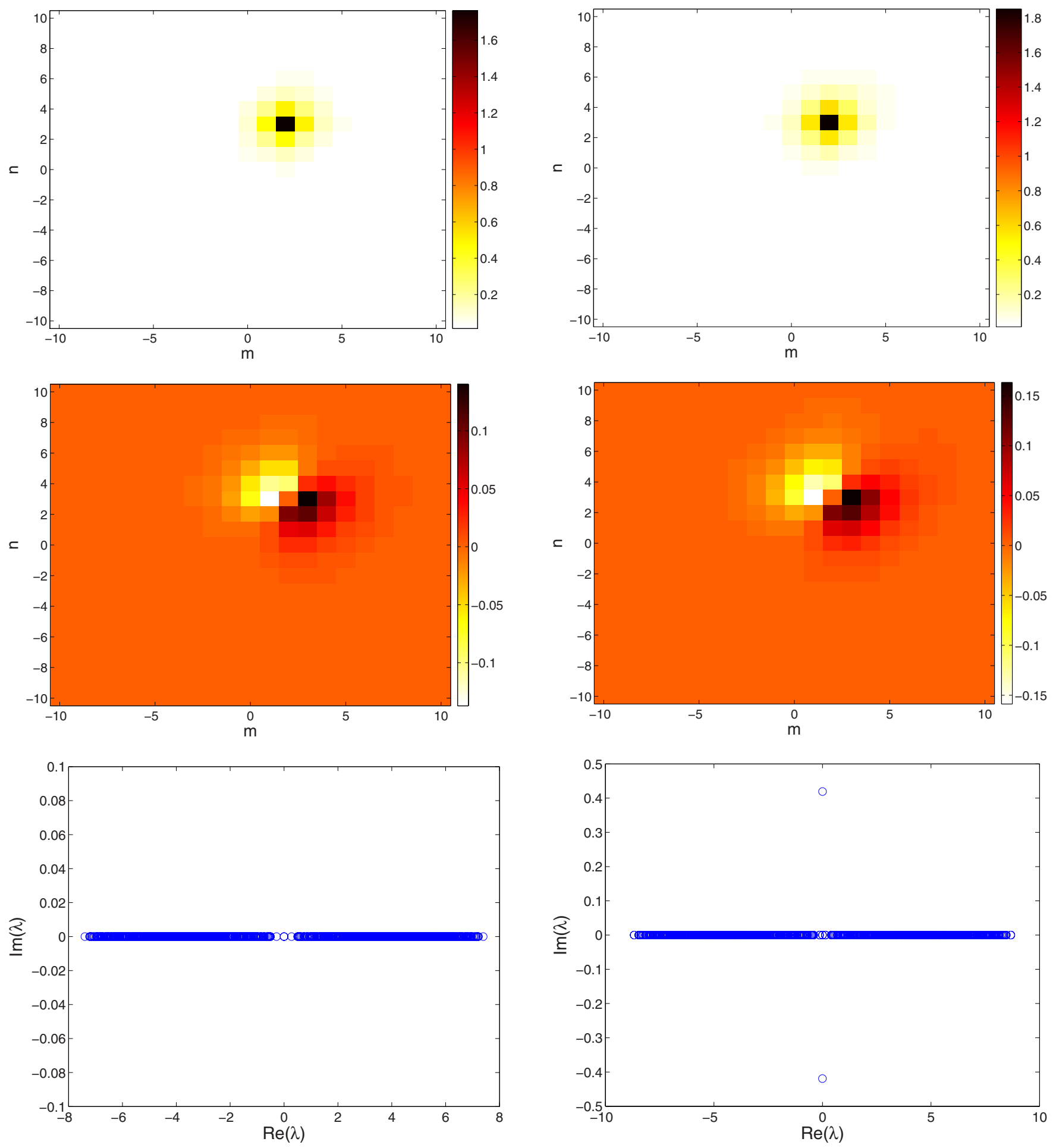

FIG. 2. (Color online) Contour plots of the real (top panel) and imaginary (middle panel) parts of a stable fundamental soliton centered at $m_{0}=3, n_{0}=2$, for $C=1.5$. Bottom panel: the complex plane of the eigenfrequencies for this soliton.

$1 / \sqrt{C}$, which is a counterpart of the lattice coupling constant in Eq. (3). Next, we assume the presence of a soliton with amplitude $A$ and intrinsic size $l$, whose center is located at distance $R$ from the rotation pivot (tantamount to the origin, in the present case). First, demanding a balance between 

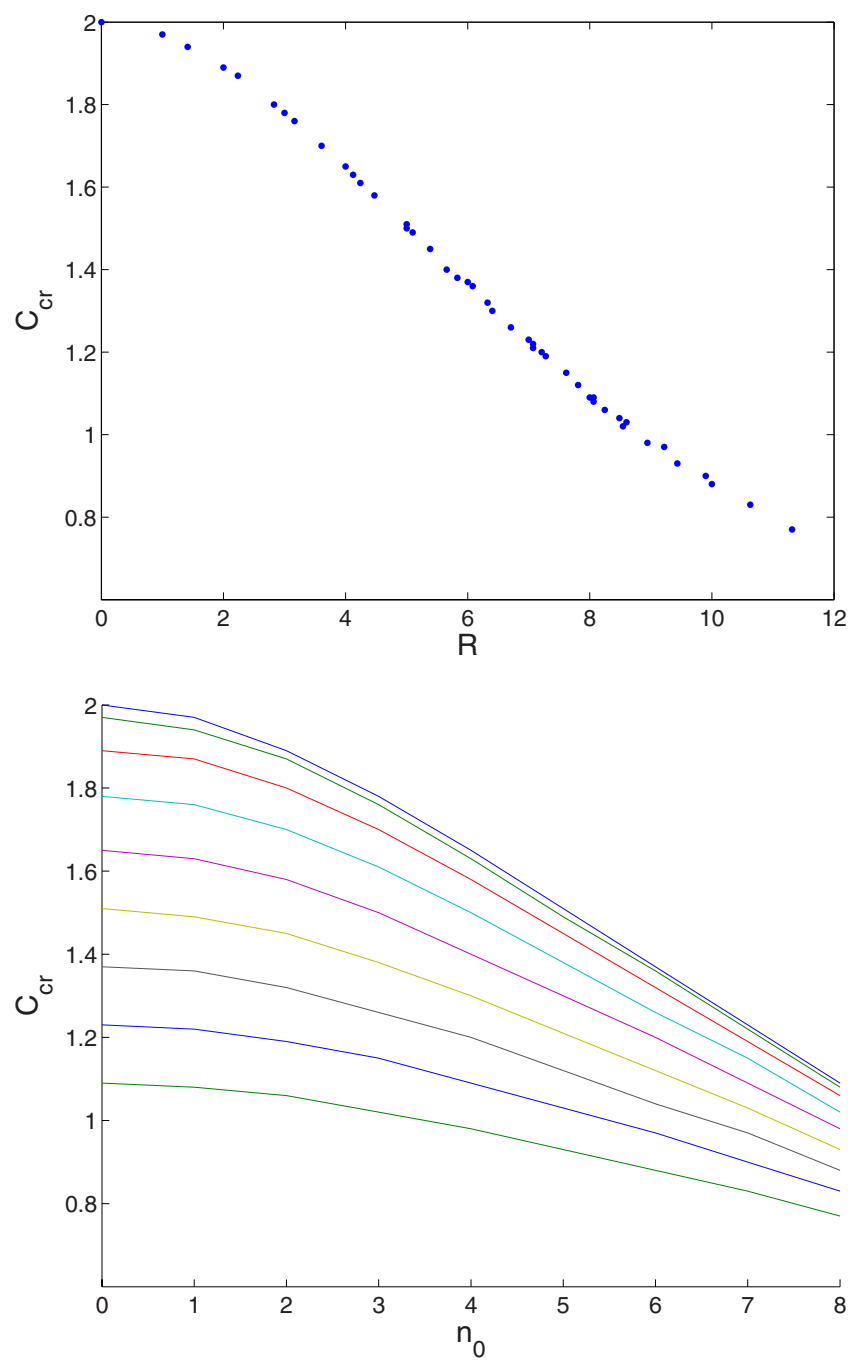

FIG. 4. (Color online) Dependence of the stability border for the fundamental solitons $C_{\mathrm{cr}}$ on the distance of the soliton's center from the rotation pivot $R$ (top panel), and on one of the center's coordinates $n_{0}$ for fixed $m_{0}$ (bottom panel). In the latter figure, the value of $n_{0} \in[0,8]$ increases progressively from top to bottom.

Further, the soliton as a whole will be in equilibrium relative to the rotating lattice potential if the action of the centrifugal force, generated by the term $\sim \Omega$ in Eq. (7), is compensated by the force of pinning to the periodic potential. The estimate of the latter condition yields $C \Omega R / l \sim \epsilon$. Substituting here $l \sim \sqrt{C /|\Lambda|}$, as per Eq. (8), we arrive at a final estimate, $R \sim(\epsilon / \sqrt{|\Lambda|} \Omega) C^{-1 / 2}$, which predicts dependence $C_{\mathrm{cr}} \sim 1 / R^{2}\left(C_{\mathrm{cr}}\right.$ is realized here as the largest value of $C$ that can provide for the balance between the centrifugal and pinning forces at given $R$ ). The latter dependence is qualitatively consistent with the numerical findings showing the decrease of $C_{\mathrm{cr}}$ with $R$ in Fig. 4 (at very large $C$, when the analytical formula predicts small $R$, it is irrelevant, as the above consideration tacitly assumed that the size of the soliton was essentially smaller than the distance to the pivot, $l$ $\ll R$; it is irrelevant too at very small $C$, as the quasicontinuum approximation cannot be used in that essentially discrete case).

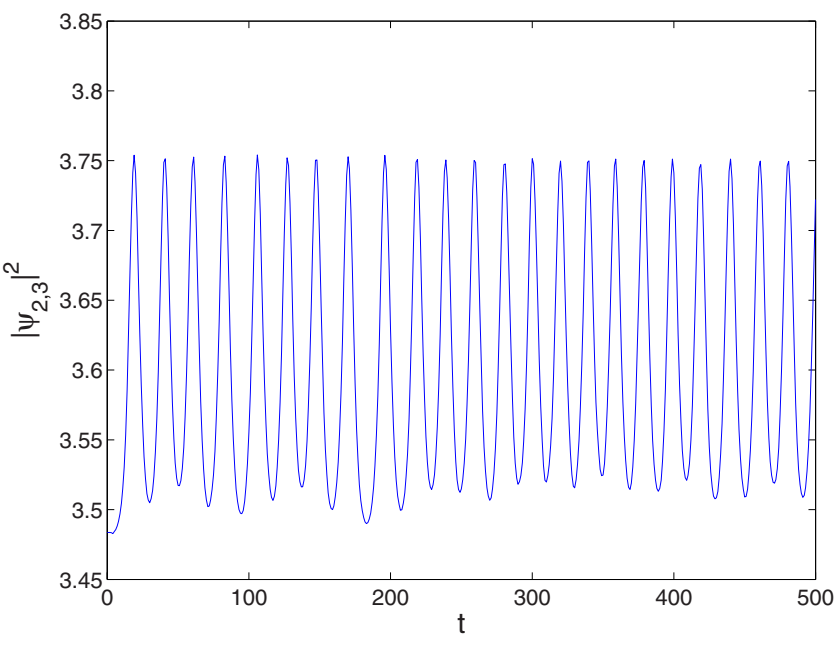

FIG. 5. (Color online) The time dependence of the squared amplitude of a perturbed unstable soliton from Fig. 3 at its center $\left(m_{0}=3, n_{0}=2\right)$. Notice the robust oscillatory behavior indicating the breathing nature of the resulting solution.

Using the quasicontinuum approximation, it is also possible to estimate the order of magnitude of the rotation frequency, in physical units. In the application to the BEC, assuming the lattice spacing $\sim 1 \mu \mathrm{m}$ and the condensate of ${ }^{7} \mathrm{Li}$ or ${ }^{85} \mathrm{Rb}$, which provide for the possibility of the attraction between atoms and, thus, the formation of solitons [31,32], and undoing of rescalings which cast the GPE in the normalized form of Eq. (1), we conclude that $\Omega=0.1$ corresponds, in physical units, to the rotation frequency $\sim 100$ or $10 \mathrm{~Hz}$, for lithium and rubidium, respectively. As concerns the above-mentioned realization of the model in terms of the twisted bundle of optical fibers, an estimate shows that, for the carrier wavelength $\sim 1 \mu \mathrm{m}$ and separation between the fibers in the bundle $\sim 10 \mu \mathrm{m}, \Omega=0.1$ corresponds to the twist pitch, which we define as a length at which the twist attains the angle of $2 \pi$, of the order of $5 \mathrm{~cm}$.

\section{VORTEX SOLITONS}

Discrete VSs of the 2D DNLS equation were systematically developed in Ref. [35] as complex stationary solutions which feature a phase circulation of $2 \pi S$ around the central point, at which the amplitude vanishes, with integer $S$ identified as the vorticity. Prior to that, time-periodic multibreather states that may feature a vortical structure were found in 2D Hamiltonian lattice dynamical models [36]. The center of the VS may coincide with a site of the lattice, or may be located in the middle of a lattice cell; the corresponding vortices are called "crosses" (alias rhombuses) and "squares," respectively. The stability of these states has been studied in detail, both for $S=1[35,37]$ and $S>1$ [29,37]. In particular, the VS crosses with $S=1$ (and, as above, with $\Lambda$ fixed to be 1) are stable in the corresponding interval of values of the coupling constant, $C<C_{\mathrm{cr}}^{(S=1)}=0.781$, and the instability above this point transforms the VS into an ordinary fundamental soliton, with $S=0$. While square VSs with $S=2$ are unstable in the ordinary 2D DNLS equation, here 
the vortex solutions with $S=3$ have their stability interval (more narrow than for $S=1$ ), $C<C_{\mathrm{cr}}^{(S=3)}=0.198$. In all cases, the instability sets in via the Hamiltonian Hopf bifurcation, represented by quartets of eigenvalues [38].

We have constructed localized vortices (of the cross or rhombus type), with $S=1$ and $S=2$, in the rotating-lattice model based on Eq. (3), and examined their stability. Unlike the above analysis of the FSs, we consider here only on-axis VSs, whose centers coincide with the rotation pivot $(R=0)$. We note that, while the FS with $R=0$ is virtually identical to its counterpart in the ordinary model, with $\Omega=0$, for VSs the situation is quite different; in particular, the VSs centered at the rotation pivot feature quite nontrivial stability properties with the increase of rotation frequency $\Omega$ (see below). Therefore, while the results for the FSs were displayed for $\Omega$ $=0.1$ and growing $R$, in this section we focus on $R=0$ but vary $\Omega$.

\section{A. Vortex solitons with $S=1$}

The first noteworthy effect of the rotation on the VS with $S=1$ is that, for given $\Omega$, its stability region features not only the upper bound $C_{\mathrm{cr}}^{(S=1)}$, as in the usual DNLS model, but also a lower one, $\widetilde{C}_{\mathrm{cr}}^{(S=1)}$

$$
\widetilde{C}_{\mathrm{cr}}^{(S=1)}<C<C_{\mathrm{cr}}^{(S=1)} .
$$

At a given value of $\Omega$, VSs are exponentially unstable for $C<\widetilde{C}_{\mathrm{cr}}^{(S=1)}$, and they feature an oscillatory instability, accounted for by a Hopf bifurcation, at $C>C_{\mathrm{cr}}^{(S=1)}$. This situation takes place for $\Omega<\Omega_{\mathrm{cr}}^{(S=1)}=0.037$. An example is given by Fig. 6 , which displays the instability growth rate of the VS $|\operatorname{Im}(\lambda)|$, versus $C$ for fixed $\Omega=0.01$. The plot also shows the real part of the eigenfrequencies, $|\operatorname{Re}(\lambda)|$, and includes, for the sake of comparison, the same dependences in the usual DNLS equation, with $\Omega=0$. The figure shows that, in this case, $\widetilde{C}_{\mathrm{cr}}^{(S=1)}=0.31$ and $C_{\mathrm{cr}}^{(S=1)}=0.788$.

The overall stability region for the VSs with $S=1$ in the plane $(C, \Omega)$ is presented in Fig. 7. It is observed that $C_{\mathrm{cr}}^{(S=1)}$ slightly increases with $\Omega$, while the growth of $\widetilde{C}_{\mathrm{cr}}^{(S=1)}$ with $\Omega$ is fast. As a result, at $\Omega>\Omega_{\mathrm{cr}}^{(S=1)}$ the stability region does not exist. In the absence of the stable VSs, unstable ones feature coexistence of exponential and Hopf instabilities. Figure 6 displays an example of the latter regime, and shows that, for $\Omega=0.04, \widetilde{C}_{\mathrm{cr}}^{(S=1)}=0.87$ and $C_{\mathrm{cr}}^{(S=1)}=0.81$.

In fact, the effect of the destabilization of the VSs with $S=1$ at small $C\left[C<\widetilde{C}_{\mathrm{cr}}^{(S=1)}\right.$, as mentioned above $]$, is a higherorder phenomenon, in terms of the expansion of the stability eigenvalues in powers of $C$, when it is assumed small. Indeed, comparing it with the stability analysis for the cross vortices in the ordinary model, with $\Omega=0$ [39], we observe the following. At $\Omega=0$, modes of small perturbations around the vortex of the cross or rhombus type feature a pair of real eigenfrequencies, with $\lambda= \pm C$ at the first order in small $C$ (in the present notation). The same pair appears in the present context (see Fig. 6). At larger $C$, these eigenfrequencies will give rise to the Hamiltonian-Hopf instability, upon their collision with eigenfrequencies bifurcating from the phonon band of linear excitations. As shown in Ref. [39], the DNLS equation with $\Omega=0$ also gives rise to a pair of higher-order eigenfrequencies, $\lambda \approx \pm C^{2}$ in the present notation. The effect of the rotation forces the latter eigenfrequencies to separate along the imaginary axis, thus inducing the instability at small $C$. However, for larger $C$, the pair again becomes real, stabilizing the configuration.

Four different regimes identified in the stability diagram in Fig. 7 are illustrated by typical examples of the stability and instability of the VSs with $S=1$ in Fig. 8 as follows: the exponential instability, caused by the higher-order eigenfrequencies, as described above, is shown in the top left panel; the top right panel shows a linearly stable case. The oscillatory instability is presented in the bottom left panel (the latter case is shown for sufficiently large $C$, to allow the eigenfrequencies, which originally linearly depend on $C$, as indicated above, to collide with eigenfrequencies bifurcating from the phonon band). Finally, the mixed oscillatory-exponential instability, which is possible at $\Omega>\Omega_{\mathrm{cr}}^{(S=1)}$, is displayed in the bottom right panel.

Since the destabilization of the VS at $C>C_{\mathrm{cr}}^{(S=1)}$ is accounted for by the Hopf bifurcation, as confirmed by Fig. 6, this unstable VS is transformed into a persistent breather (not shown here), which loses the vortical structure, i.e., is similar to the FS [35]. On the other hand, the destabilization at $C$ $<\widetilde{C}_{\mathrm{cr}}^{(S=1)}$ occurs, as seen in Fig. 6, via a pair of imaginary eigenfrequencies with zero real parts, i.e., an exponential instability. Its nonlinear development eventually leads to a persistently pulsating localized state with zero vorticity, as illustrated by Fig. 9. The transformation to a FS state is also observed in the region of the coexistence of exponential and oscillatory instabilities.

\section{B. Vortex solitons with $S=2$}

Another way in which the rotating lattice drastically alters the stability features of the ordinary 2D DNLS model concerns the VSs with $S=2$. At $\Omega=0$, all square localized vortices with $S=2$ are unstable due to an imaginary eigenfrequency proportional (at the leading order) to coupling constant $C[35,37]$, see the top panel of Fig. 10 (which may be compared with Fig. 4 of [37]). In the rotating lattice, the solitons with $S=2$ acquire a finite stability region, as manifested by the example displayed in Fig. 10. Note that, unlike the stability interval for the VSs with $S=1$, see Eq. (9), only an upper stability border exists for the $S=2$ solitons, i.e., the respective stability interval is $0<C<C_{\mathrm{cr}}^{(S=2)}$. For instance, in the case shown in Fig. 10, the stability border induced by the rotation is $C_{\mathrm{cr}}^{(S=2)}=0.12$. Note that the mechanism of the stabilization of the $S=2 \mathrm{VSs}$ in the present model is different from that reported in Ref. [40], where localized vortices with $S=2$ were stabilized by an impurity (inert site) placed at the center. In that case, the unstable eigenmode was suppressed by the defect, making all eigenfrequencies real; eventual destabilization occurred due to collisions of those real eigenfrequencies with the linear spectrum. We should also note that very recently rhombic vortices of $S=2$ were proposed [41] and were found to be linearly stable for small $C$. Here, the rotation affects the unstable (imaginary) eigenfrequency 

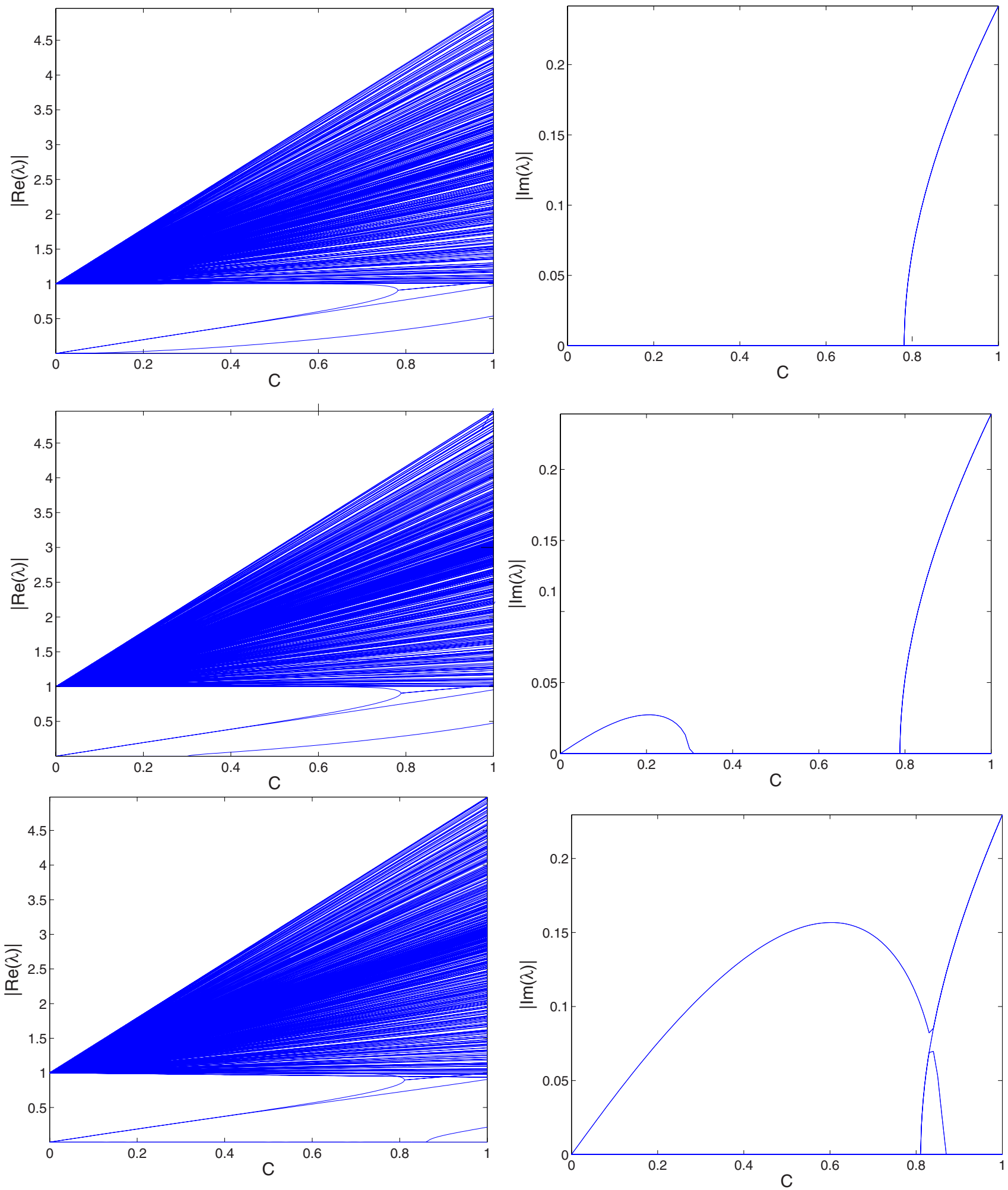

FIG. 6. (Color online) Dependences on $C$ of the real and imaginary parts (left and right panels, respectively) of eigenfrequencies of small perturbations about vortex solitons with $S=1$ in the ordinary (nonrotating) DNLS lattice (top panels), and in the rotating one, with $\Omega$ $=0.01$ (central panels) and $\Omega=0.04$ (bottom panels). In the rotating case, the center of the vortex coincides with the rotation pivot of the lattice.

of the $S=2 \mathrm{VS}$, rendering it real for small $C$. However, as $C$ is increased the eigenfrequency eventually becomes imaginary again, leading to the instability of the VS.
The stability diagram for the VSs with $S=2$ in the $(C, \Omega)$ plane is presented in Fig. 11, indicating the increasing stabilization effect of larger rotation frequencies. As in the case of 


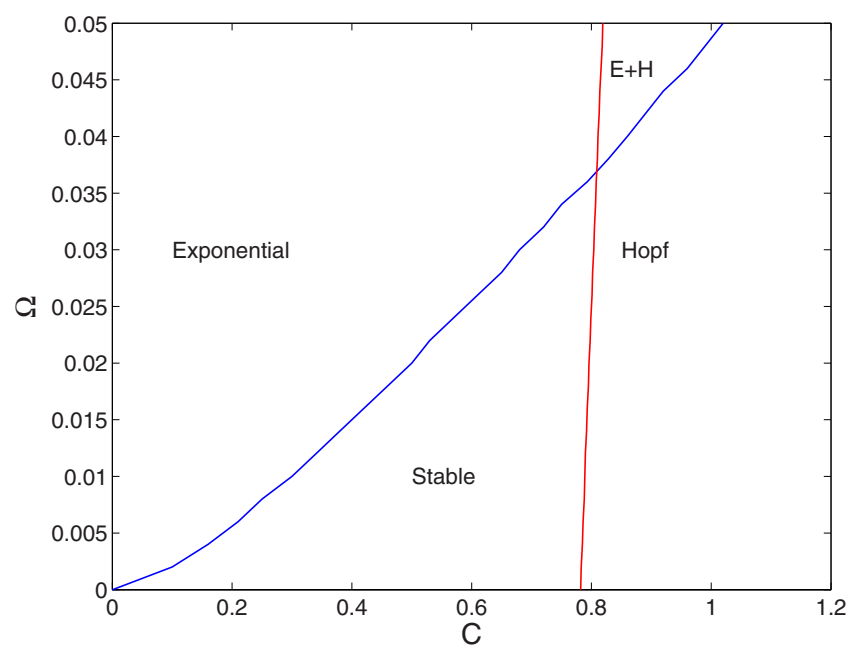

FIG. 7. (Color online) The full stability diagram for vortex solitons with $S=1$, centered at the rotation pivot. The $E+H$ label holds for coexisting Hopf and exponential instabilities.
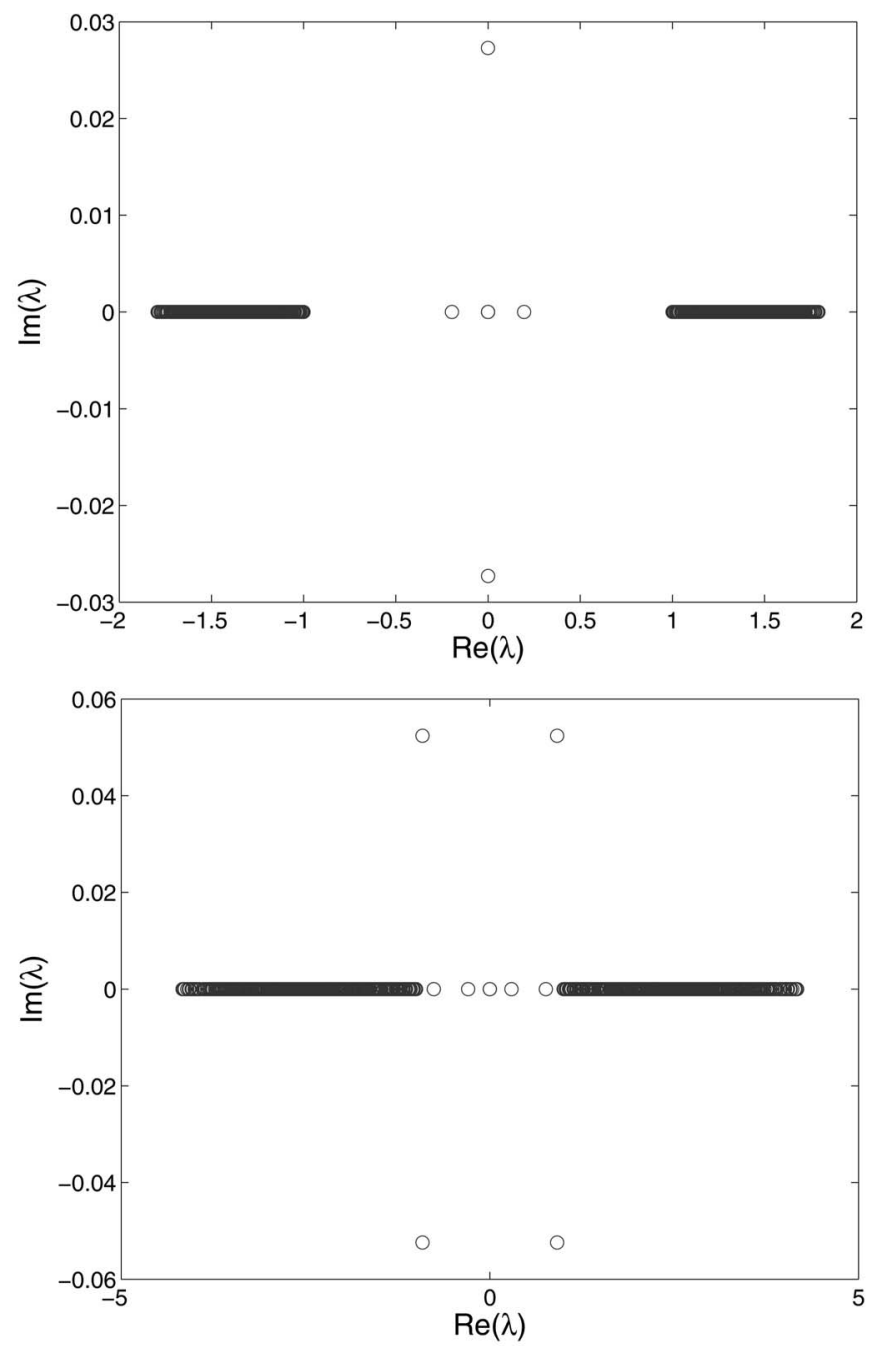

$S=1$, the instabilities of the VSs with $S=2$ transform it into a persistent breather, but without the vortical structure. In fact, a similar qualitative conclusion was made in the ordinary model, with $\Omega=0$.

\section{Quadrupole and octupole solitons}

Along with complex solutions for localized vortices with $S \geq 2$, the ordinary 2D DNLS equation, with $\Omega=0$, gives rise to real solutions in the form of quadrupoles and octupoles, that resemble higher-order vortices, but carry no topological charge [25,29]. In particular, quadrupoles, which include four lattice sites with alternating phases, have their stability region $0<C \leq C_{\mathrm{cr}}^{\text {(quad) }}$ in the model with $\Omega=0$; the same is true for octupoles, which are based on eight sites with alternating phases. In the anticontinuum limit, a case example of the quadrupole and octupole solutions may be seeded, respectively, by the following configurations: $\phi_{0, \pm 1}=1, \phi_{ \pm 1,0}$ $=-1, \quad$ and $\quad \phi_{0,2}=\phi_{1,-1}=\phi_{2,1}=\phi_{-1,0}=1, \phi_{1,2}=\phi_{0,-1}=\phi_{-1,1}$ $=\phi_{2,0}=-1$, all other sites having $\phi_{m, n}=0$. Note that the latter
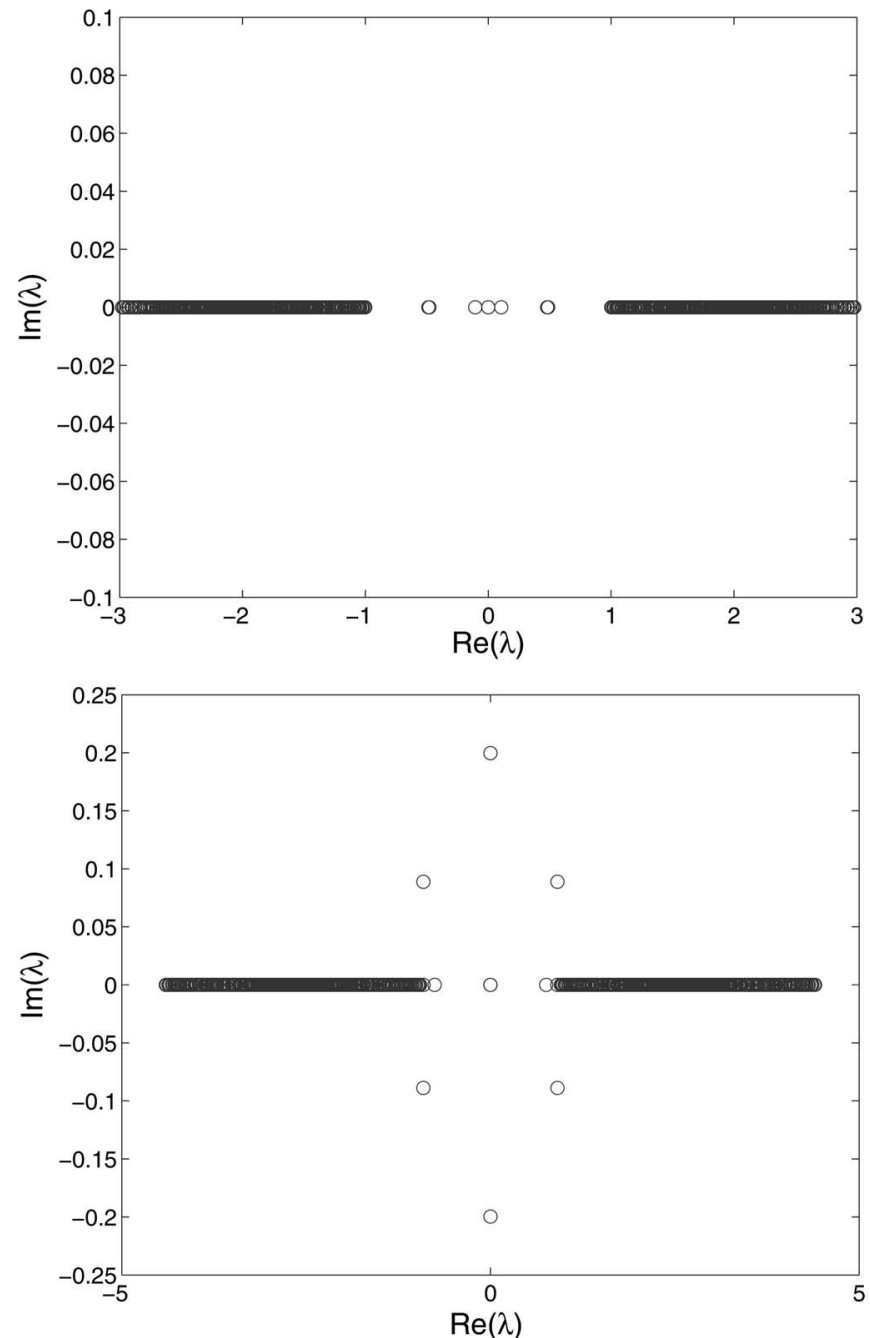

FIG. 8. Eigenfrequencies of small perturbations around the vortex with $S=1$ are shown for the four different cases: exponential instability for $\Omega=0.01$ and $C=0.2$ (top left panel); linear stability at $\Omega=0.01$ and $C=0.5$ (top right panel); oscillatory instability at $\Omega=0.01$ and $C$ $=0.8$ (bottom left panel); and exponential and oscillatory instability at $\Omega=0.05$ and $C=0.85$ (bottom right panel). 


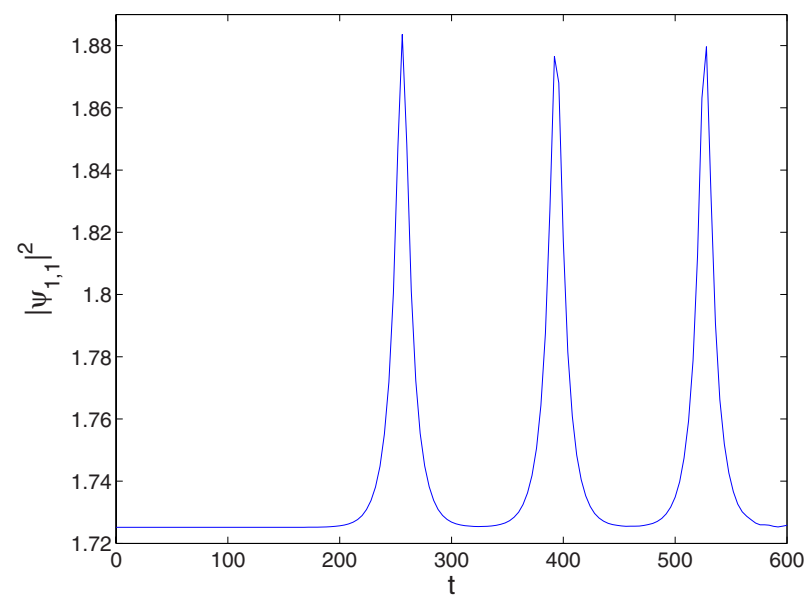

FIG. 9. (Color online) The time dependence of the amplitude of a perturbed unstable vortex soliton with $S=1, \Omega=0.03$, and $C$ $=0.4$. Note that the point corresponding to this soliton is located in the left instability region in Fig. 7, i.e., the vortex is unstable to nonoscillatory perturbations (also see the text).
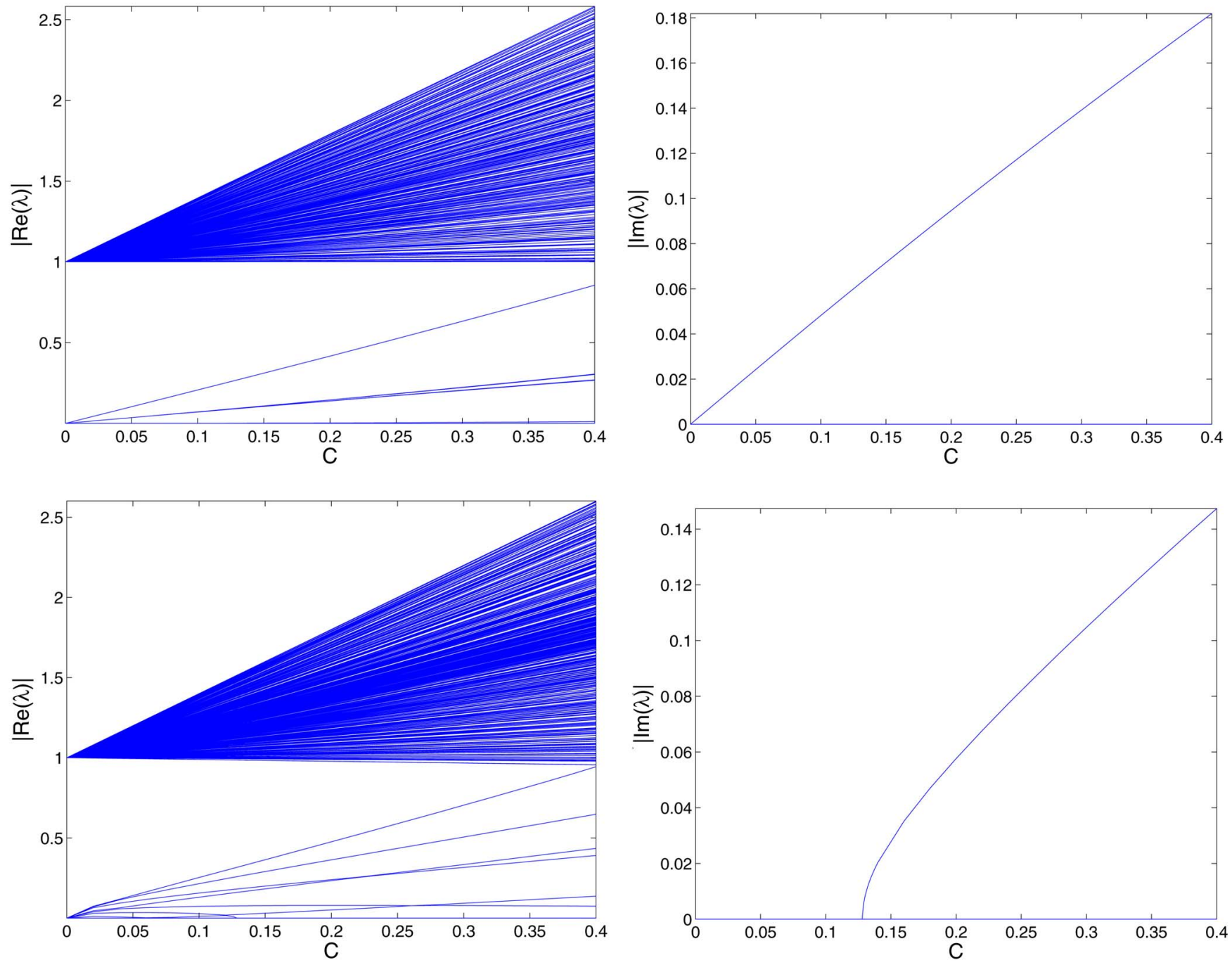

FIG. 10. (Color online) The same as in Fig. 6, but for the vortex solitons with $S=2$. Top and bottom panels correspond to $\Omega=0$ and $\Omega=0.05$, respectively. Notice the contrast between the absence of instabilities at small $C$ in the bottom right panel and the immediate destabilization in the top right panel. 


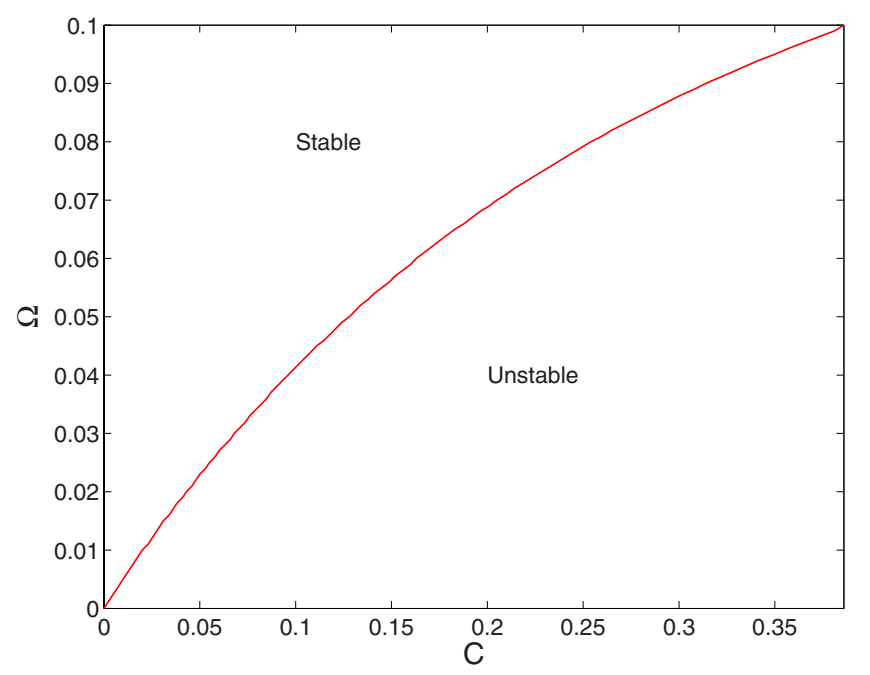

FIG. 11. (Color online) The stability diagram, similar to Fig. 7, but for vortex solitons with $S=2$.

cubic nonlinearity and rotating square-lattice potential. The discrete model can be implemented in BEC stirred by a rotating strong optical lattice, or, in principle, also in a twisted bundle of nonlinear optical fibers. Localized solutions of two types were considered: off-axis FSs, with the center placed at distance $R$ from the rotation pivot, and on-axis VSs, with vorticity $S=1$ and 2 . For the FSs, the stability interval was found in the form of $0<C<C_{\mathrm{cr}}(R)$, where $C$ is the coupling constant of the discrete lattice, and $C_{\mathrm{cr}}(R)$ a monotonically decreasing function (see Fig. 4). A qualitative explanation to this result was proposed, based on the analysis of the balance between the lattice-pinning and centrifugal forces. For VSs with $S=1$, the dependence of the stability region on the rotation frequency $\Omega$ was found, in the form of Eq. (9) and Fig. 7 , with the conclusion that the stability is only possible for $\Omega<\Omega_{\mathrm{cr}}$. A key feature, which makes the situation different from earlier stability analyses of such structures in the standard 2D DNLS model, is a higher-order eigenfrequency, shifted toward instability for sufficiently weak lattice coupling, in the presence of rotation. On the other hand, VSs with $S=2$, which are always unstable in the model with $\Omega$ $=0$, are stabilized by the rotation in the region $0<C$
$<C_{\mathrm{cr}}^{(S=2)}$, as shown in Fig. 11. For the vortices with $S=2$, the reverse effect happens in comparison with $S=1$, namely, an unstable (at $\Omega=0$ ) eigenfrequency is tipped by the rotation in the opposite direction; i.e., for $S=1$ a real eigenfrequency becomes imaginary in the presence of $\Omega$, while the reverse is true for $S=2$. Quadrupole and octupole solitons, with the center coinciding with the pivotal point, were briefly considered too, with a conclusion that their stability regions are almost the same as in the ordinary model (with $\Omega=0$ ). An estimate for relevant values of $\Omega$ in physical units was given for both physical realizations of the model, i.e., the rotation frequency of the optical lattice stirring the self-attractive BEC, and the pitch of the twisted bundle of optical fibers.

The analysis initiated in this work can be developed in several directions. In particular, results obtained in the continuum model considered in Ref. [26] suggest that, at sufficiently large $\Omega$, the fully localized FSs, with the center shifted off the axis (i.e., continuum counterparts of the discrete FSs considered in the present work), are unstable (or do not exist), while stable ring-shaped solitons may appear instead, with the center of the ring coinciding with the pivot.

Another interesting direction would be to apply techniques elaborated on in Refs. $[37,18]$ to this considerably more difficult setting. It would be especially relevant to repeat the calculation of the eigenfrequencies for the vortices with $S=1$ and $S=2$ by means of those methods in the presence of rotation, and quantify the impact of $\Omega$ on the eigenvalues, to rigorously investigate some effects which were outlined above in a qualitative form.

Another possibility is to consider a discrete limit of the model with the rotating quasi-1D potential, such as the one with the repulsive cubic nonlinearity, which was introduced in Ref. [27]. Studies along these directions are currently underway and will be reported elsewhere.

\section{ACKNOWLEDGMENTS}

J.C. acknowledges financial support from the MECD Project No. FIS2004-01183. The work of B.A.M. was partially supported by the Israel Science Foundation through Excellence-Center Grant No. 8006/03, and by German-Israel Foundation through Grant No. 149/2006. P.G.K. gratefully acknowledges the support of NSF-DMS-0505663, NSFDMS-0619492, and NSF-CAREER.
[1] D. N. Christodoulides and R. I. Joseph, Opt. Lett. 13, 794 (1988).

[2] H. S. Eisenberg, Y. Silberberg, R. Morandotti, A. R. Boyd, and J. S. Aitchison, Phys. Rev. Lett. 81, 3383 (1998).

[3] S. Darmanyan, A. Kobyakov, E. Schmidt, and F. Lederer, J. Exp. Theor. Phys. 86, 682 (1998); P. G. Kevrekidis, A. R. Bishop, and K. Ø. Rasmussen, Phys. Rev. E 63, 036603 (2001); A. A. Sukhorukov and Y. S. Kivshar, ibid. 65, 036609 (2002).

[4] A. Maluckov, M. Stepić, D. Kip, and L. Hadzievski, Eur. Phys. J. B 45, 539 (2005).
[5] N. K. Efremidis, S. Sears, D. N. Christodoulides, J. W. Fleischer, and M. Segev, Phys. Rev. E 66, 046602 (2002).

[6] J. W. Fleischer, G. Bartal, O. Cohen, T. Schwartz, O. Manela, B. Freedman, M. Segev, H. Buljan, and N. K. Efremidis, Opt. Express 13, 1780 (2005); A. S. Desyatnikov, N. Sagemerten, R. Fischer, B. Terhalle, D. Träger, D. N. Neshev, A. Dreischuh, C. Denz, W. Krolikowski, and Y. S. Kivshar, ibid. 14, 2851 (2006).

[7] J. W. Fleischer, T. Carmon, M. Segev, N. K. Efremidis, and D. N. Christodoulides, Phys. Rev. Lett. 90, 023902 (2003); D. Neshev, E. Ostrovskaya, Y. Kivshar, and W. Krolikowski, Opt. 
Lett. 28, 710 (2003).

[8] J. W. Fleischer, M. Segev, N. K. Efremidis, and D. N. Christodoulides, Nature (London) 422, 147 (2003).

[9] D. N. Neshev, T. J. Alexander, E. A. Ostrovskaya, Y. S. Kivshar, H. Martin, I. Makasyuk, and Z. G. Chen, Phys. Rev. Lett. 92, 123903 (2004); J. W. Fleischer, G. Bartal, O. Cohen, O. Manela, M. Segev, J. Hudock, and D. N. Christodoulides, ibid. 92, 123904 (2004).

[10] A. Szameit, D. Blömer, J. Burghoff, T. Schreiber, T. Pertsch, S. Nolte, A. Tünnermann, and F. Lederer, Opt. Express 13, 10552 (2005).

[11] A. Szameit, Y. V. Kartashov, F. Dreisow, T. Pertsch, S. Nolte, A. Tünnermann, and L. Torner, Phys. Rev. Lett. 98, 173903 (2007).

[12] X. Wang, A. Bezryadina, Z. Chen, K. G. Makris, D. N. Christodoulides, and G. I. Stegeman, Phys. Rev. Lett. 98, 123903 (2007).

[13] A. Trombettoni and A. Smerzi, Phys. Rev. Lett. 86, 2353 (2001); F. Kh. Abdullaev, B. B. Baizakov, S. A. Darmanyan, V. V. Konotop, and M. Salerno, Phys. Rev. A 64, 043606 (2001); A. Smerzi, A. Trombettoni, P. G. Kevrekidis, and A. R. Bishop, Phys. Rev. Lett. 89, 170402 (2002); R. CarreteroGonzález and K. Promislow, Phys. Rev. A 66, 033610 (2002).

[14] G. L. Alfimov, P. G. Kevrekidis, V. V. Konotop, and M. Salerno, Phys. Rev. E 66, 046608 (2002).

[15] F. S. Cataliotti, S. Burger, C. Fort, P. Maddaloni, F. Minardi, A. Trombettoni, A. Smerzi, and M. Inguscio, Science 293, 843 (2001).

[16] M. Greiner, O. Mandel, T. Esslinger, T. W. Hänsch, and I. Bloch, Nature (London) 415, 39 (2002).

[17] P. G. Kevrekidis, B. A. Malomed, D. J. Frantzeskakis, and R. Carretero-González, Phys. Rev. Lett. 93, 080403 (2004); R. Carretero-González, P. G. Kevrekidis, B. A. Malomed, and D. J. Frantzeskakis, ibid. 94, 203901 (2005).

[18] M. Lukas, D. Pelinovsky, and P. G. Kevrekidis, e-print arXiv:nlin/0610070, Phys. Rev. D (to be published).

[19] J. E. Heebner and R. W. Boyd, J. Mod. Opt. 49, 2629 (2002); P. Chak, J. E. Sipe, and S. Pereira, Opt. Lett. 28, 1966 (2003).

[20] J. J. Baumberg, P. G. Savvidis, R. M. Stevenson, A. I. Tartakovskii, M. S. Skolnick, D. M. Whittaker, and J. S. Roberts, Phys. Rev. B 62, R16247 (2000); P. G. Savvidis and P. G. Lagoudakis, Semicond. Sci. Technol. 18, S311 (2003).

[21] P. G. Kevrekidis, K. Ø. Rasmussen, and A. R. Bishop, Int. J. Mod. Phys. B 15, 2833 (2001).

[22] B. B. Baizakov, B. A. Malomed, and M. Salerno, Europhys. Lett. 63, 642 (2003); J. K. Yang and Z. H. Musslimani, Opt.
Lett. 28, 2094 (2003).

[23] B. B. Baizakov, B. A. Malomed, and M. Salerno, Phys. Rev. A 70, 053613 (2004).

[24] W. J. Firth and D. V. Skryabin, Phys. Rev. Lett. 79, 2450 (1997).

[25] H. Sakaguchi and B. A. Malomed, Europhys. Lett. 72, 698 (2005).

[26] H. Sakaguchi and B. A. Malomed, Phys. Rev. A 75, 013609 (2007).

[27] Y. V. Kartashov, B. A. Malomed, and L. Torner, Phys. Rev. A 75, 061602(R) (2007).

[28] V. Schweikhard, I. Coddington, P. Engels, S. Tung, and E. A. Cornell, Phys. Rev. Lett. 93, 210403 (2004); R. Bhat, L. D. Carr, and M. J. Holland, ibid. 96, 060405 (2006); S. Tung, V. Schweikhard, and E. A. Cornell, ibid. 97, 240402 (2006); K. Kasamatsu and M. Tsubota, ibid. 97, 240404 (2006).

[29] P. G. Kevrekidis, B. A. Malomed, Z. Chen, and D. J. Frantzeskakis, Phys. Rev. E 70, 056612 (2004); P. G. Kevrekidis, H. Susanto, and Z. Chen, ibid. 74, 066606 (2006).

[30] J. Gómez-Gardeñes, L. M. Floría, and A. R. Bishop, Physica D 216, 31 (2006).

[31] K. E. Strecker, G. B. Partridge, A. G. Truscott, and R. G. Hulet, Nature (London) 417, 150 (2002); L. Khaykovich, F. Schreck, G. Ferrari, T. Bourdel, J. Cubizolles, L. D. Carr, Y. Castin, and C. Salomon, Science 256, 1290 (2002).

[32] S. L. Cornish, S. T. Thompson, and C. E. Wieman, Phys. Rev. Lett. 96, 170401 (2006).

[33] T. Pertsch, U. Peschel, and F. Lederer, Phys. Rev. E 66, 066604 (2002).

[34] J. Yang, I. Makasyuk, P. G. Kevrekidis, H. Martin, B. A. Malomed, D. J. Frantzeskakis, and Z. Chen, Phys. Rev. Lett. 94, 113902 (2005).

[35] B. A. Malomed and P. G. Kevrekidis, Phys. Rev. E 64, 026601 (2001).

[36] S. Aubry, Physica D 103, 201 (1997); T. Cretegny and S. Aubry, ibid. 113, 162 (1998); M. Johansson, S. Aubry, Y. B. Gaididei, P. L. Christiansen, and K. Ø. Rasmussen, ibid. 119, 115 (1998).

[37] D. E. Pelinovsky, P. G. Kevrekidis, and D. J. Frantzeskakis, Physica D 212, 20 (2005).

[38] J.-C. van der Meer, Nonlinearity 3, 1041 (1990).

[39] P. G. Kevrekidis and D. E. Pelinovsky, Proc. R. Soc. London, Ser. A 462, 2671 (2006).

[40] P. G. Kevrekidis and D. J. Frantzeskakis, Phys. Rev. E 72, 016606 (2005).

[41] M. Öster and M. Johansson, Phys. Rev. E 73, 066608 (2006). 